\title{
Paralisia nervosa na artroplastia total do quadril ${ }^{*}$
}

\author{
Nerve palsy in total hip replacement
}

\author{
Carlos Roberto Schwartsmann ${ }^{1}$, Antony Kerbes Yepez ${ }^{2}$
}

\section{RESUMO}

A paralisia nervosa pós-prótese total do quadril é uma complicação incomum $(0,17$ a $1 \%)$. A presente revisão, após levantamento bibliográfico sobre 0 assunto, analisa 50 artigos relevantes sobre 0 tema. A revisão tem como objetivos analisar dados da fisiopatologia, prevalência, etiologia, fatores de risco, prognóstico e prevenção dessa complicação.

Descritores - Quadril; Artroplastia de quadril; Complicações pós-operatórias

\section{ABSTRACT}

Nerve palsy after total hip replacement is a rare complication $(0.17 \%$ to $1 \%)$. This review of the literature on this topic reviews 50 relevant papers on the issue. The purpose of the review is to analyze pathophysiology, prevalence, etiology, risk factors, prognosis, and prevention of this complication.

\section{Keywords - Hip; Hip arthroplasty; Postoperative complications}

* Trabalho realizado no Complexo Hospitalar Santa Casa de Porto Alegre (RS), Brasil.

1. Professor Titular de Ortopedia da Fundação Faculdade Federal de Ciências Médicas de Porto Alegre (RS), Brasil. Chefe do Serviço de Ortopedia e Traumatologia do Complexo Hospitalar Santa Casa de Porto Alegre (RS), Brasil.

2. Estagiário do Grupo de Quadril do Complexo Hospitalar Santa Casa de Porto Alegre (RS), Brasil.

Endereço para correspondência: Prof. Dr. Carlos Roberto Schwartsmann, Rua Leopoldo Bier, 825 - Bairro Santana - 90620-100 - Porto Alegre, RS. Fone: (51) 3214-8074 (manhã) - (51) 3217-6422 (tarde) - tel/fax (51) 3214-8285.

E-mail: schwartsmann@santacasa.tche.br.

Copyright RBO2008

\section{INTRODUÇÃO}

Paralisia nervosa é uma complicação incomum pósartroplastia total do quadril, mas, quando ocorre, o impacto desse acontecimento pode ser imperceptível ou até mesmo devastador. A relação médico-paciente deteriora-se rapidamente à medida que o tempo passa $\mathrm{e}$ não existem sinais de recuperação. $O$ paciente não compreende essa ocorrência nestes dias de quase perfeição.

$\mathrm{O}$ presente artigo tem como objetivo revisar a literatura e oferecer aos ortopedistas, e até mesmo a juristas, conhecimentos e subsídios sobre fisiopatologia, prevalência, etiologia, fatores de risco, prognóstico e prevenção dessa complicação.

\section{FISIOPATOLOGIA}

A gravidade da lesão nervosa periférica foi classificada por Seddon ${ }^{(1)}$ de acordo com o grau de lesão anatômica.

A neurapraxia é uma paralisia funcional do nervo sem lesão anatômica da estrutura neurológica. Há perda da condução nervosa, geralmente devida a compressão, contusão ou isquemia.

A axonotmese ocorre na presença de lesão axonal com degeneração mielínica, mas sem rotura do tubo endoneural.

Em ambos os casos, a função nervosa pode ser restabelecida.

A neurotmese ocorre quando há completa secção do tubo neural com permanente perda nervosa funcional. A função nervosa só pode ser restabelecida após reparação cirúrgica.

\section{PREVALÊNCIA}

Nercessian et al, revisando mais de 7.000 artroplastias, calcularam que a prevalência de paralisia nervosa 
no membro superior após prótese total do quadril é de aproximadamente $0,15 \%{ }^{(2)}$. Essa lesão geralmente está associada a problemas relacionados à posição do paciente na mesa cirúrgica e ocorre mais freqüentemente quando o diagnóstico primário é de artrite reumatóide.

Paralisias do membro inferior contralateral também são raras. Smith et al relataram cinco casos em 919 $\operatorname{artroplastias}^{(3)}$. Em geral, são relacionados também ao posicionamento do paciente.

Na maioria das vezes, a paralisia ocorre no membro operado e é devida a trauma direto ou indireto, compressão ou distração.

$\mathrm{Na}$ tentativa de avaliar com fidedignidade a prevalência, foram incluídos 38 artigos na presente revisão.

A menor prevalência de paralisia nervosa foi de 0,08\% (uma paralisia em 1.287 casos) ${ }^{(4)}$.

A maior prevalência foi de 7,6\% (cinco paralisias em 66 revisões de artroplastias) ${ }^{(5)}$.

A tabela 1 mostra a prevalência da paralisia nervosa associada a artroplastia total do quadril em 38 artigos.

No total de 70.134 artroplastias foram registradas 529 paralisias. O percentual correspondente é de $0,75 \%$.

\section{ETIOLOGIA E FATORES DE RISCO}

Sexo feminino - Weber et al ${ }^{(17)}$, Solheim et al $^{(20)}$, Johanson et $a l^{(23)}$, Edwards et al ${ }^{(27)}$, Navarro et $a l^{(33)}$ encontraram prevalência muito maior no sexo feminino. Isso provavelmente deve-se ao fato de que as mulheres, quando comparadas com os homens, são menores em altura e têm membros menores associados a menor massa muscular e, portanto, mais suscetíveis à lesão nervosa periférica. Avaliando seis estudos, Schmalzried et $l^{(30)}$ concluíram que as mulheres possuem duas vezes mais risco de evoluir com paralisia pós-prótese total do quadril que os homens.

Cirurgia de revisão - Schmalzried et al ${ }^{(30)}$ avaliaram cinco estudos que compararam paralisia nervosa em prótese primária ou de revisão. Concluíram que a artroplastia de revisão tem risco três vezes maior. Obviamente, isso se explica pela alteração da anatomia, presença de aderências, necessidade de melhor exposição e, muitas vezes, alongamento do membro.
TABELA 1

Prevalência de lesão nervosa associada com artroplastia total do quadril

\begin{tabular}{|c|c|c|c|c|}
\hline Autor & Ano & $\begin{array}{l}\text { Número } \\
\text { de casos }\end{array}$ & $\begin{array}{c}\text { Número } \\
\text { de lesões }\end{array}$ & $\%$ \\
\hline Stern et $a l^{(6)}$ & 1972 & 50 & 2 & 4,0 \\
\hline Charnley et $a I^{(7)}$ & 1973 & 185 & 2 & 1,1 \\
\hline Evarts et $a l^{(8)}$ & 1973 & 200 & 6 & 3,0 \\
\hline Langenskiold et al(9) & 1973 & 116 & 1 & 0,9 \\
\hline Wilson et $a l^{(10)}$ & 1973 & 108 & 4 & 3,7 \\
\hline Lubinus $^{(11)}$ & 1973 & 1.350 & 28 & 2,1 \\
\hline Lazansky $^{(12)}$ & 1973 & 501 & 6 & 1,2 \\
\hline Buchholz et al(13) & 1973 & 3.948 & 76 & 1,9 \\
\hline Eftekhar et $a I^{(14)}$ & 1973 & 800 & 12 & 1,5 \\
\hline Murray ${ }^{(15)}$ & 1973 & 808 & 15 & 1,9 \\
\hline Smith ${ }^{(16)}$ & 1973 & 112 & 1 & 0,9 \\
\hline Weber et $a /^{(17)}$ & 1976 & 2.012 & 14 & 0,7 \\
\hline Moczynski et al(18) & 1976 & 237 & 3 & 1,3 \\
\hline Beckenbaugh et al(19) & 1978 & 300 & 4 & 1,3 \\
\hline Sarmiento et $a /^{(25)}$ & 1979 & 237 & 7 & 3,0 \\
\hline Solheim et $a I^{(20)}$ & 1980 & 825 & 6 & 0,7 \\
\hline Robinson et al (21) & 1980 & 316 & 2 & 0,6 \\
\hline Amstutz et $a l^{(5)}$ & 1982 & 66 & 5 & 7,6 \\
\hline Tillberg ${ }^{(22)}$ & 1982 & 327 & 3 & 0,9 \\
\hline Johanson et $a I^{(23)}$ & 1983 & 5.667 & 36 & 0,6 \\
\hline Ahlgren et $a I^{(24)}$ & 1984 & 150 & 1 & 0,7 \\
\hline Andrew et $a l^{(26)}$ & 1985 & 179 & 1 & 0,6 \\
\hline Edwards et $a l^{(27)}$ & 1987 & 614 & 7 & 1,1 \\
\hline Soballe et $a I^{(28)}$ & 1987 & 213 & 1 & 0,5 \\
\hline Mok et al (29) & 1989 & 251 & 4 & 1,6 \\
\hline Schmalzried et $a l^{(30)}$ & 1991 & 3.126 & 53 & 1,7 \\
\hline Simmons et $a I^{(31)}$ & 1991 & 440 & 10 & 2,3 \\
\hline Dhillon et al(32) & 1992 & 380 & 6 & 1,6 \\
\hline Nercessian et $a I^{(2)}$ & 1994 & 7.133 & 45 & 0,63 \\
\hline Nercessian et $a l^{(4)}$ & 1994 & 1.287 & 1 & 0,08 \\
\hline Navarro et al $I^{(33)}$ & 1995 & 1.000 & 8 & 0,8 \\
\hline Linclau et $a /^{(34)}$ & 1995 & 1.463 & 7 & 0,5 \\
\hline Oldenburg et al(35) & 1997 & 2.723 & 61 & 2,2 \\
\hline Pekkarinen et $a l^{(36)}$ & 1999 & 4.339 & 27 & 0,6 \\
\hline Eggli et al(37) & 1999 & 508 & 8 & 1,6 \\
\hline Butt et $a I^{(38)}$ & 2005 & 355 & 6 & 1,7 \\
\hline Farrell et al ${ }^{(39)}$ & 2005 & 27.004 & 47 & 0,17 \\
\hline Hurd et $a l^{(40)}$ & 2006 & 804 & 3 & 0,37 \\
\hline Total & & 70.134 & 529 & 0,75 \\
\hline
\end{tabular}

Fonte: pesquisa realizada em artigos publicados no Pubmed.

Diagnóstico inicial - O risco de paralisia ocorrer no diagnóstico de displasia do quadril é maior, pois a anatomia normal é alterada, bem como a relação das estruturas nervosas ${ }^{(23,30,39,41)}$. Como, na maioria dos casos há necessidade de alongamento do membro inferior, o risco se torna mais elevado ainda. 
Alongamento do membro inferior - Johanson et al ${ }^{(23)}$, Edwards et $a l^{(27)}$, Shaughnessy et $a l^{(41)}$ referem que alongamento de mais de $4 \mathrm{~cm}$ durante a artroplastia está diretamente associado a paralisia.

Edwards et $a l^{(27)}$ reportaram casos de paralisia do nervo peroneiro após alongamentos de $2,7 \mathrm{~cm}$ e paralisia do nervo ciático após alongamentos de $4,4 \mathrm{~cm}$.

Nercessian $e t a l^{(2)}$ advogaram que a percentagem de alongamento do membro é mais importante que um número absoluto. A percentagem de alongamento não deve ultrapassar $15 \%$ do comprimento do mesmo.

Lunborg ${ }^{(42)}$, estudando o efeito da distração nervosa com microangiografia, concluiu que a circulação cessa após alongamento de $15 \%$ do membro inferior.

Via de acesso - Não está claro que a via de acesso pode influenciar a ocorrência de paralisia ${ }^{(21,30,33)}$.

Evidentemente, algumas lesões nervosas podem ser resultantes de compressão direta por afastadores. Portanto, a lesão do nervo femoral ocorre mais frequientemente no acesso ântero-lateral e a lesão do nervo ciático, mais no acesso posterior.

Simmons et $a l^{(31)}$ relataram sete paralisias do nervo femoral em 360 artroplastias operadas pelo acesso ântero-lateral. Estudo anatômico demonstrou a vulnerabilidade do nervo por afastadores na parede e rebordo anterior do acetábulo.

Mallory ${ }^{(43)}$ descreveu um caso de lesão ciática que ocorreu após englobamento do nervo na cerclagem do trocanter após via de acesso de Charnley. Ramesh et $a l^{(44)}$ estudaram clínica e eletromiograficamente $81 \mathrm{ca}-$ sos operados pelo acesso lateral direto (Hardinge). Após duas semanas, encontraram 19 (23\%) pacientes com lesão do nervo glúteo superior e evidente fraqueza dos abdutores.

Farrell et al $^{(39)}$, analisando 27.004 artroplastias, consideraram estatisticamente significante o risco de paralisia ciática quando utilizado o acesso posterior.

Formação de hematoma - A formação de hematoma pode ocasionar paralisia tardia no pós-operatório imediato devida a excessiva anticoagulação para profilaxia da trombose venosa ${ }^{(23,30,39,45-46)}$.

Quando o hematoma envolve o compartimento glúteo, o nervo ciático pode ser lesado ${ }^{(45,47)}$. Quando o he- matoma envolve o músculo ilíaco, o nervo femoral pode ser atingido ${ }^{(46,48)}$.

Prótese cimentada - Vários casos de paralisia pósprótese total do quadril cimentado são descritos ocasionados pelo extravasamento do cimento. Ele pode ocorrer pela parede medial ou pela parede posterior do acetábulo, ocasionando paralisia femoral ou ciática, respectivamente ${ }^{(30,39,48-49)}$.

Prótese não cimentada - Vários artigos alertam que o uso de parafusos para fixar o acetábulo não cimentado pode ocasionar paralisias ${ }^{(2,30,36,39,50)}$.

Essa preocupação originou o sistema de quatro quadrantes descrito por Wasielewski et al ${ }^{(50)}$. Os parafusos só podem ser colocados nos quadrantes pósterosuperior e póstero-inferior do acetábulo.

Farrell et $a l^{(39)}$ foram os únicos a comprovar estatisticamente que a prótese não cimentada aumenta o risco da ocorrência de paralisia ciática. Entretanto, colocam em dúvida essa relação, pois na maioria dos seus casos foi usada também a via de acesso posterior.

Outros fatores de risco - Entre outros fatores de risco são incluídos: luxação da prótese, compressão do nervo peroneiro na cabeça do perônio, migração de enxerto, migração da prótese, etc.

Contudo, vale salientar que os fatores etiológicos não são conclusivos ou claros em mais de $50 \%$ dos $\operatorname{casos}^{(2,13,17,30,35,39,49)}$.

\section{PROGNÓSTICO}

Em relação ao prognóstico, apesar da frustração inicial, ele deve ser encarado com visão otimista. Segundo Schmalzried et al ${ }^{(30)}$, a recuperação completa ocorre em aproximadamente $41 \%$ dos casos; $44 \%$ podem evoluir com déficit neurológico menor e aproximadamente $15 \%$ têm prognóstico reservado, com fraqueza, deambulação limitada e persistente disestesia. Em geral, a recuperação do nervo femoral é mais previsível que a do nervo ciático. É lógico que o prognóstico depende do grau de lesão nervosa.

Inúmeros autores sugerem que a recuperação do nervo femoral seria melhor que a do nervo ciático, pelo fato de a regeneração nervosa necessitar de distância 
menor para ocorrer no primeiro do que no segundo ${ }^{(20-}$ 27-31-39-48).

Outros autores não têm uma visão tão otimista: Pekkarinen et $a l^{(36)}$, acompanhando 27 casos, concluíram que a recuperação total ocorreu em oito $(29,7 \%)$, a recuperação parcial em sete $(25,9 \%)$ e déficit permanente, em 12 (44,4\%).

Farrell et $a^{(39)}$, seguindo 28 paralisias, concluíram que $10(35,7 \%)$ recuperaram-se integralmente. A recuperação parcial ocorreu em sete $(25,0 \%)$ e o déficit permanente, em 11 casos $(39,3 \%)$.

Se o paciente apresentar algum grau de recuperação em duas semanas, o prognóstico se torna mais favorável. Recuperação consistente ocorre no primeiro ano, mas a regeneração nervosa pode acontecer em três $\operatorname{anos}^{(2-30-36-39)}$.

\section{PREVENÇÃO}

Com base na revisão de literatura, a prevalência de lesão nervosa pós-artroplastia total de quadril é de $0,75 \%$.

Deve-se levar em conta que esses relatos de lesões nervosas pós-prótese total do quadril são originados de grandes centros médicos especializados, experientes e interessados no assunto. A verdadeira incidência de paralisias nervosas na comunidade ortopédica geral provavelmente é bem maior.

$\mathrm{O}$ risco de paralisia nervosa associada à artroplastia total de quadril aumenta significativamente nas mulheres, no diagnóstico de displasia do quadril e nas cirurgias de revisão. Esses são os três principais fatores de risco.

Na maioria dos casos, é muito difícil identificar com clareza a causa da paralisia nervosa. Se foi por excessiva tração do membro, compressão por afastadores, alongamento excessivo, por parafuso ou por lesão térmica do cimento, etc.

Quando a paralisia ocorre e é diagnosticada, é de boa prática discutir a complicação o mais rapidamente possível com o paciente e familiares. Uma formal consulta com neurologista deve ser realizada, pois se trata de uma lesão nervosa periférica.

A decisão de reoperar deve ser muito cautelosa e o fator etiológico deve ser bem evidente: presença de hematoma, compressão por parafuso ou enxerto ósseo, excessivo alongamento, etc.

A documentação dessa ocorrência e da evolução dos fatos deve ser precisa e obsessiva. Cuidadosos exames clínicos seqüenciais com estudos de condução nervosa devem ser realizados. Cabe ao ortopedista tentar manter a relação com o paciente da maneira mais cordial, clara e confiável possível, pois o tratamento dessa complicação é longo e freqüentemente a relação médico-paciente se deteriora.

No entender do paciente, de uma forma ou de outra, independente do fator etiológico, o médico tem culpa: como é possível submeter-se a uma cirurgia para resolver um problema e após a mesma o problema se tornar maior? Muitas vezes, a situação se torna insustentável, há quebra do relacionamento e a questão extrapola a esfera médica e envolve a esfera jurídica. Por isso, a prevenção da paralisia e o tratamento são preferíveis.

Evidentemente que a prevenção está associada ao profundo conhecimento anatômico da região e à técnica cuidadosa de dissecção. Deve-se evitar o uso de afastadores com pontas finas ou cortantes. Os auxiliares devem ser orientados para afastar com elegância e sem usar força excessiva. Evitar torções extremas do membro inferior. Manter sempre que possível o joelho em flexão porque reduz a tensão sobre o nervo ciático. Evitar colocação de parafusos nos quadrantes de risco. Nas próteses cimentadas, evitar perfurações acetabulares e extravamento do cimento. Na ocorrência de perfuração acidental, é necessário tamponar o pertuito com enxerto ósseo da cabeça. Atenção na manipulação da cerclagem, das suturas profundas para não envolver nervos.

\section{CONCLUSÕES}

Revisando 50 artigos sobre paralisia nervosa na artroplastia total do quadril, concluímos que a prevalência é de $0,75 \%$.

A melhor maneira de tratar essa complicação é evitá-la.

Desconhecer a anatomia regional, os fatores de risco e os inúmeros fatores etiológicos aumenta as probabilidades da ocorrência dessa desagradável complicação. 


\section{REFERÊNCIAS}

1. Seddon HJ. Three types of nerve injury. Brain. 1943;(66):23788 .

2. Nercessian OA, Macaulay W, Stinchfield FE. Peripheral neuropathies following total hip replacement. J Arthroplasty. 1994;9(6):645-51.

3. Smith JW, Pellicci PM, Sharrock N, Mineo R, Wilson Jr PD. Complications after total hip replacement. The contralateral limb. J Bone Joint Surg Am. 1989;71(4):528-35.

4. Nercessian OA, Piccoluga F, Eftekhar NS. Postoperative sciatic and femoral nerve palsy with reference to leg lengthening and medialization/lateralization of the hip joint following total hip arthroplasty. Clin Orthop Relat Res. 1994;(304):165-71.

5. Amstutz HC, Ma SM, Jinnah RH, Mai L. Revision of aseptic loose total hip arthroplasties. Clin Orthop Relat Res. 1982; (170):21-33.

6. Stern MB, Grant SS. Fifty total hip replacements. An initial experience. Clin Orthop Relat Res. 1972;(86):79-84.

7. Charnley J, Cupic Z. The nine and ten year results of the lowfriction arthroplasty of the hip. Clin Orthop Relat Res. 1973; (95):9-25.

8. Evarts CM, DeHaven KE, Nelson CL, Collins HR, Wilde AH. Interim results of Charnley-Muller total hip arthroplasty. Clin Orthop Relat Res. 1973;(95):193-200.

9. Langenskiold A, Paavilainen T. Total hip replacement of 116 hips by the McKee-Farrar prosthesis. A preliminary report. Clin Orthop Relat Res. 1973;(95):143-50.

10. Wilson JN, Scales JT. The Stanmore metal on metal total hip prosthesis using a three pin type cup. A follow-up of 100 arthroplasties over nine years. Clin Orthop Relat Res. 1973; (95):239-49.

11. Lubinus HH. Total hip replacement using the "BrunswikSystem”. Clin Orthop Relat Res. 1973;(95):211-2.

12. Lazansky MG. Complications revisited. The debit side of total hip replacement. Clin Orthop Relat Res. 1973;(95):96-103.

13. Buchholz HW, Noack G. Results of the total hip prosthesis design "St. George". Clin Orthop Relat Res. 1973;(95):201-10.

14. Eftekhar NS, Stinchfield FE. Experience with low-friction arthroplasty. A statistical review of early results and complications. Clin Orthop Relat Res. 1973;(95):60-8.

15. Murray WR. Results in patients with total hip replacement arthroplasty. Clin Orthop Relat Res. 1973;(95):80-90.

16. Smith RD. Total hip replacement: metal against metal. Review and analysis of cases - 1961-1972. Clin Orthop Relat Res. 1973;(95):43-7.

17. Weber ER, Daube JR, Coventry MB. Peripheral neuropathies associated with total hip arthroplasty. J Bone Joint Surg Am. 1976;58(1):66-9.

18. Moczynski G, Abraham E, Barmada R, Ray RD. Evaluation of total hip replacement arthroplasties. Clin Orthop Relat Res. 1976;(95):213-6.
19. Beckenbaugh RD, Ilstrup DM. Total hip arthroplasty. J Bone Joint Surg Am. 1978;60(3):306-13.

20. Solheim LF, Hagen R. Femoral and sciatic neuropathies after total hip replacement. Acta Orthop Scand. 1980;51(3):531-4.

21. Robinson RP, Robinson Jr HJ, Salvati EA. Comparison of the transtrochanteric and posterior approaches for total hip replacement. Clin Orthop Relat Res. 1980;(147):143-7.

22. Tillberg B. Total hip arthroplasty using the McKee and WatsonFarrar prosthesis. A prospective follow-up study of 327 arthroplasties. Acta Orthop Scand. 1982;53(1):103-7.

23. Johanson NB, Pellicci PM, Tsairis P, Salvati EA. Nerve injury in total hip arthroplasty. Clin Orthop Relat Res. 1983;(179): 214-22.

24. Ahlgren SA, Elmqvist D, Ljung P. Nerve lesions after total hip replacement. Acta Orthop Scand. 1984;55(2):152-5.

25. Sarmiento A, Zych GA, Latta LL, Tarr RR. Clinical experiences with a titanium alloy total hip prothesis: a posterior approach. Clin Orthop Relat Res. 1979;(144):166-73.

26. Andrew TA, Berridge D, Thomas A, Duke RN. Long-term review of ring total arthroplasty. Clin Orthop Relat Res. 1985; (201):111-22.

27. Edwards BN, Tullos HS, Noble PC. Contributory factors and etiology of sciatic nerve palsy in total hip replacement. Clin Orthop Relat Res. 1987;(218):136-41.

28. Soballe K, Olsen NJ, Ejsted R, Christensen F, Luxhoj T. Revision of the uncemented hip prosthesis. Acta Orthop Scand. 1987;58(6):630-3.

29. Mok DW, Bryant KM. Ring uncemented plastic-on-metal hip replacements: results from an independent unit. J R Soc Med. 1989;82(3):142-4.

30. Schmalzried TP, Amstutz HC, Dorey FJ. Nerve palsy associated with total hip replacement. Risk factors and prognosis. J Bone Joint Surg Am. 1991;73(7):1074-80.

31. Simmons Jr C, Izant TH, Rothman RH, Booth Jr RE, Balderston RA. Femoral neuropathy following total hip arthroplasty. Anatomic study, case reports and literature review. J Arthroplasty. 1991;6(Suppl):S57-S66.

32. Dhillon MS, Nagi ON. Sciatic nerve palsy associated with total hip arthroplasty. Ital J Orthop Traumatol. 1992;18(4):521-6.

33. Navarro RA, Schmalzried TP, Amstutz HC, Dorey FJ. Surgical approach and nerve palsy in total hip arthroplasty. J Arthroplasty. 1995;10(1):1-5.

34. Linclau L, Dokter G, Gutwirth P. Postoperative dropfoot after cementless total hip arthroplasty. Acta Orthop Belg. 1995;61(4): 271-7.

35. Oldenburg M, Müller RT. The frequency, prognosis and significance of nerve injuries in total hip arthroplasty. Int Orthop. 1997;21(1):1-3.

36. Pekkarinen J, Alho A, Puusa A, Paavilainen T. Recovery of sciatic nerve injuries in association with total hip arthroplasty in 27 patients. J Arthroplasty. 1999;14(3):305-11. 
37. Eggli S, Hankemayer S, Muller ME. Nerve plasy after leg lengthening in total replacement arthroplaty for developmental dysplasia of the hip. J Bone Joint Surg Br. 1999;81(5):843-5.

38. Butt AJ, McCarthy T, Kelly IP, Glynn T, McCoy G. Sciatic nerve palsy secondary to postoperative haematoma in primary total hip replacement. J Bone Joint Surg Br. 2005;87(11):1465-7.

39. Farrell CM, Springer BD, Haidukewych GJ, Morrey BF. Motor nerve palsy following primary total hip arthroplasty. J Bone Joint Surg Am. 2005;87(12):2619-25.

40. Hurd JL, Potter HG, Dua V, Ranawat CS. Sciatic nerve plasy after primary total hip arthroplasty: a new perspective. J Arthroplasty. 2006;21(6):796-802.

41. Shaughnessy WJ, Kavanagh B, Fitzgerald RH Jr. Long-term results of total hip arthroplasty in patients with high congenital dislocation of the hip. Orthop Trans. 1989;(13):510-2.

42. Lundborg G. Structure and function of the intraneural microvessels as related to trauma edema formation and nerve function. J Bone Joint Surg Am. 1975;57(7):938-48.

43. Mallory TH. Sciatic nerve entrapment secondary to trochanteric wiring following total hip replacement. A case report. Clin Orthop Relat Res. 1983;(180):198-200.
44. Ramesh M, O’Byrne JM, McCarthy N, Jarvis A, Mahalingham $\mathrm{K}$, Cashman WF. Damage to the superior gluteal nerve after the Hardinge approach to the hip. J Bone Joint Surg Br. 1996;78(6): 903-6.

45. Fleming RE, Michelsen CB, Stinchfield FE. Sciatic paralysis. A complication of bleeding following hip surgery. J Bone Joint Surg Am. 1979;61(1):37-9.

46. Brantigan JW, Owens ML, Moody FG. Femoral neuropathy complicating anticoagulant therapy. Am J Surg. 1976;132(1): 108-9.

47. Cohen B, Bhamra M, Ferris BD. Delayed sciatic nerve palsy following total hip arthroplasty. Br J Clin Pract. 1991;45(4): 292-3.

48. Lewallen DG. Neurovascular injury associated with hip arthroplasty. Instr Course Lect. 1998;47:275-83. Review.

49. Oleksak M, Edge AJ. Compression of the sciatic nerve by methylmethacrylate cement after total hip replacement. J Bone Joint Surg Br. 1992;74(5):729-30.

50. Wasielewski RC, Crossett LS, Rubash HE. Neural and vascular injury in total hip arthroplasty. Orthop Clin North Am. 1992; 23(2):219-35. 